\title{
Analysis of NIST Monoclonal Antibody Reference Material Glycosylation using LC-MS/MS-Based Glycoproteomic Approach
}

1 Department of Chemistry and Biochemistry, Texas Tech University, Lubbock, TX 79409

Jingfu Zhao ${ }^{\S 1}$, Wenjing Peng ${ }^{\S 1}$, Xue Dong ${ }^{1}$ and Yehia Mechref*1

§These authors contributed equally

*Corresponding Author

Department of Chemistry and Biochemistry

Texas Tech University

Lubbock, TX 79409-1061

Email: yehia.mechref@ttu.edu

Tel: 806-742-3059

Fax: 806-742-1289 


\section{Table of Contents}

Table S1. List of NISTmAb Fc glycan structures that were identified using CID-MS/MS. Symbols: blue square denotes for $\mathrm{N}$-acetylglucosamine; green circle denotes for mannose; yellow circle denotes for galactose; red triangle denotes for fucose; pale diamond denotes for $\mathrm{N}$-glycolylneraminic acid (NeuGc).

Table S2. Glycopeptides identification and their relative abundances. Putative glycan structures attached on $\mathrm{N} 300$ site with different peptide backbones resulted from miscleavages of tryptic digestion. Nomenclature of glycans: $h$ denotes for the number of hexose (Hex), $n$ denotes the number of hexosamine (HexNAc), $f$ denotes the number of fucose (DeoxyHex), a denotes the number of N-acetylneraminic acid (NeuAc), g denotes the number of N-glycolylneraminic acid (NeuGc). For example, h5n4flg2 denotes the glycan $\mathrm{Hex}_{5} \mathrm{HexNAc}_{4}$ DeoxyHex $\mathrm{NeuGc}_{2}$. (This table is provided as an Excel file) 
Table S1. List of NISTmAb Fc glycan structures that were identified using CID-MS/MS. Symbols: blue square denotes for $\mathrm{N}$-acetylglucosamine; green circle denotes for mannose; yellow circle denotes for galactose; red triangle denotes for fucose; pale diamond denotes for N-glycolylneraminic acid (NeuGc).

\begin{tabular}{|c|c|c|c|c|}
\hline Hexose & HexNAc & Fucose & NeuGc & Glycan Structure \\
\hline 2 & 3 & 1 & 0 & g-0-0 \\
\hline 3 & 2 & 1 & 0 & $\Delta=0$ \\
\hline 3 & 3 & 0 & 0 & $\left.=-\alpha_{0}^{0}\right\}=$ \\
\hline 3 & 3 & 1 & 0 & {$\left[\begin{array}{l}0 \\
0\end{array} \mid=\right.$} \\
\hline 3 & 4 & 0 & 0 & -matan \\
\hline 3 & 4 & 1 & 0 & $\cos =$ \\
\hline 3 & 5 & 1 & 0 & \\
\hline 4 & 3 & 1 & 0 & 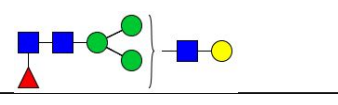 \\
\hline 4 & 3 & 0 & 1 & $=\square-0_{0}^{0} \mid-\infty$ \\
\hline 4 & 3 & 1 & 1 & $a+\infty \mid-\infty$ \\
\hline 4 & 4 & 1 & 0 & $\operatorname{ar} 0_{0}^{\infty}=0$ \\
\hline 4 & 4 & 1 & 1 & $\therefore a_{0}^{\infty}=\infty$ \\
\hline 5 & 2 & 0 & 0 & - \\
\hline 5 & 3 & 1 & 1 & 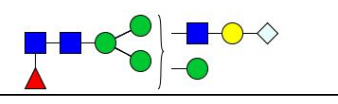 \\
\hline 5 & 4 & 1 & 0 & 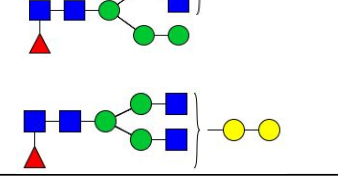 \\
\hline 5 & 4 & 1 & 1 & $\boldsymbol{\Delta}=0$ \\
\hline
\end{tabular}









\section{Figure Legend}

Figure S1. Identification of unglycosylated and deglycosylated peptides EEQYNSTYR. (A) Extracted ion chromatogram (EIC) of unglycosylated peptide EEQYNSTYR $([\mathrm{M}+\mathrm{H}]$ $\left.{ }^{+}=1189.5120,[\mathrm{M}+2 \mathrm{H}]^{2+}=595.2596\right)$ and deglycosylated peptide EEQYDSTYR $\left([\mathrm{M}+\mathrm{H}]^{+}=1190.4960,[\mathrm{M}+2 \mathrm{H}]^{2+}=595.7516\right)$. (B) MS2 spectrum and fragment ions of unglycosylated peptide at $19.5 \mathrm{~min}$. (C) MS2 spectrum and fragment ions of deglycosylated peptide at $21.5 \mathrm{~min}$. Abundant $\mathrm{y}$ and $\mathrm{b}$ fragment ions are highlighted with green and purple colors, where green color denotes the same fragment $\mathrm{m} / \mathrm{z}$ between (B) and (C), from y1 to y4, while purple color denotes an 1 Da shift between (B) and (C) due to the change of $\mathrm{N}$ to $\mathrm{D}$ in deglycosylated peptide, from y5 to $\mathrm{y} 7$.

Figure S2. Comparison of galactosylation, sialylation, fucosylation, afucosylation, and high-mannose levels in the two NIST samples. Error bars reprent standard deviation (SD); $* * * *$ indicates a statistical significance of $\mathrm{p} \leq 0.0001$ determined by student's $t$-test.

Figure S3. The EIC of oxonium ion $\mathrm{m} / \mathrm{z} 204.0867$ (GlcNAc) in SID full MS. The multiple EIC peaks of $\mathrm{m} / \mathrm{z} 204.0867$ indicated the presence of glycopeptide precursors at different retention times caused by the difference in either types of glycans or length of peptide backbones. 
A

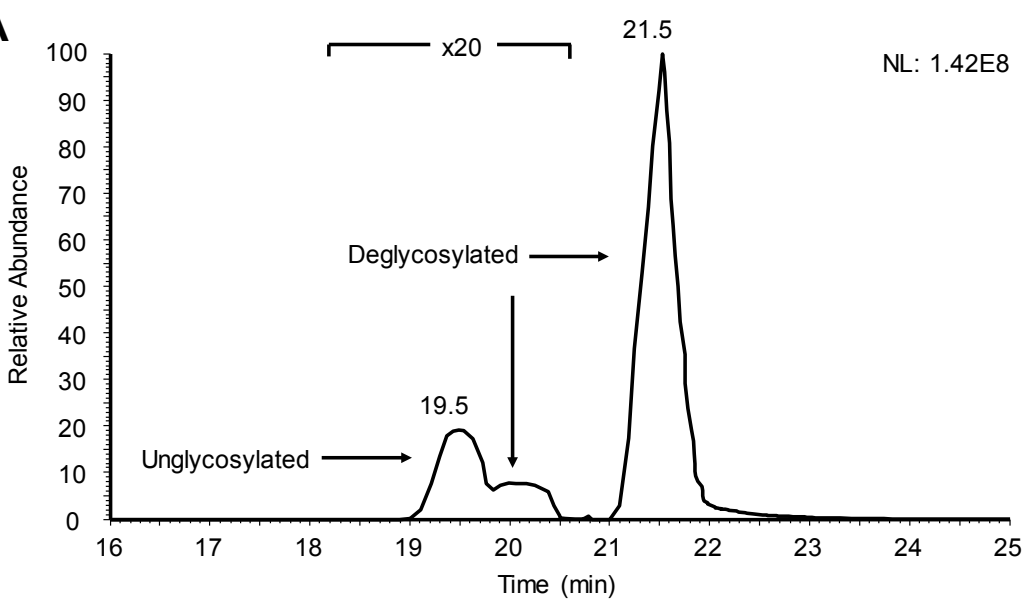

B
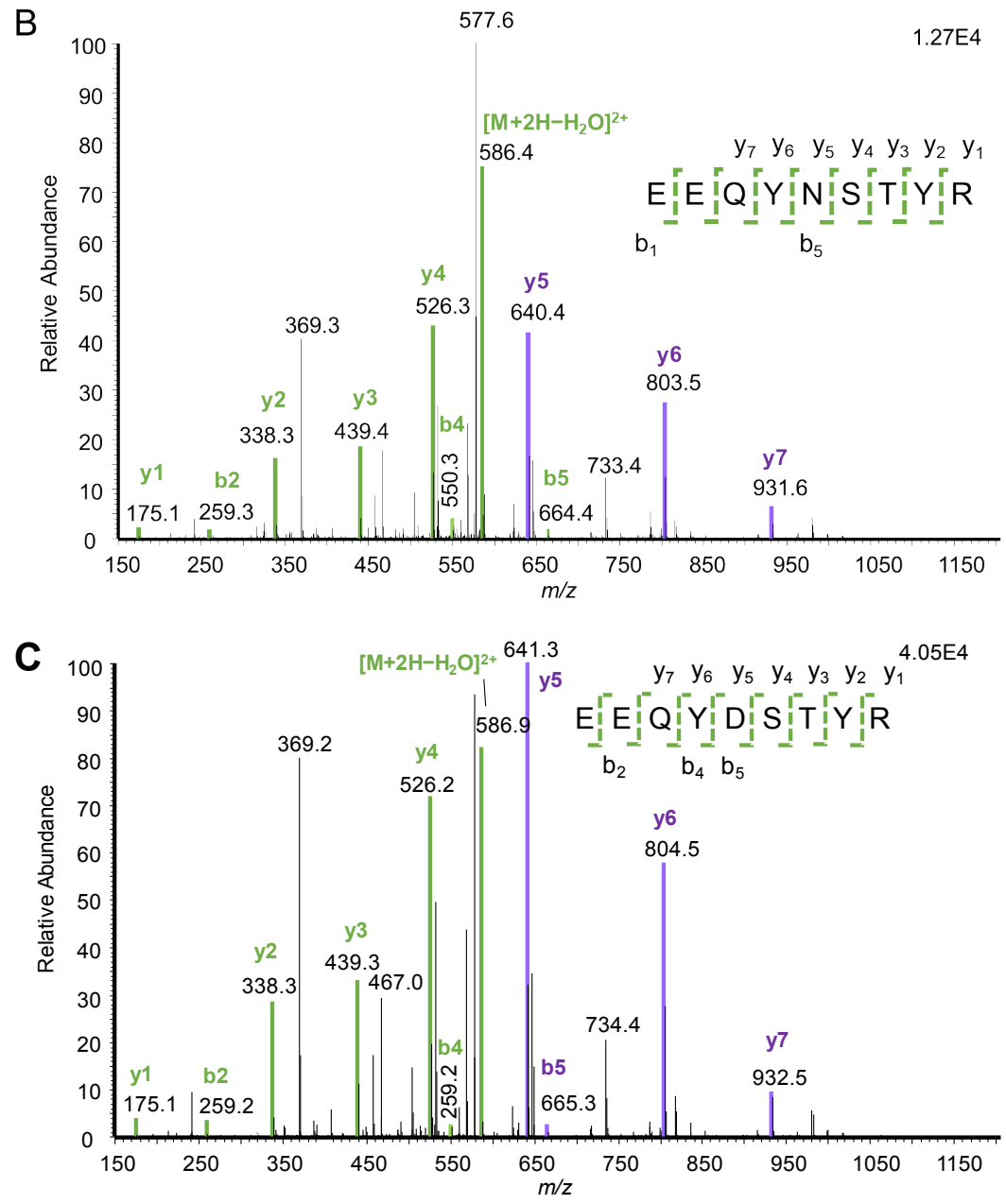
Zhao et al. Figure S2

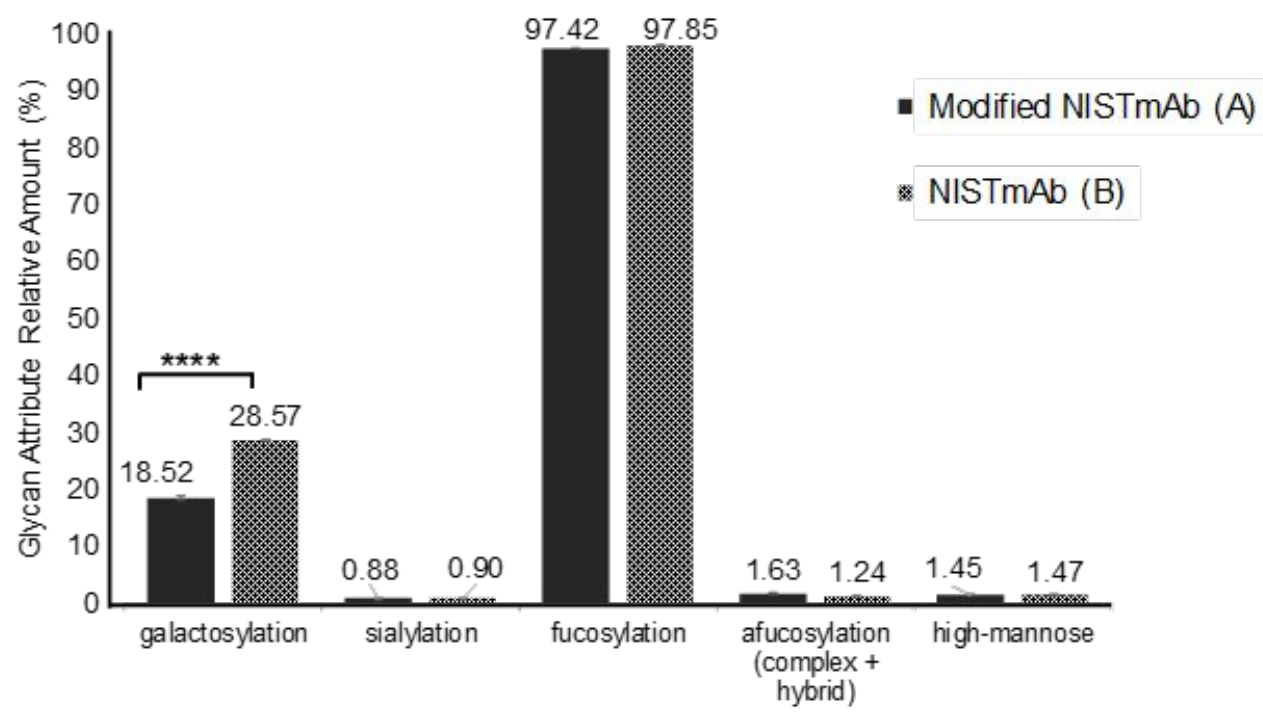






